\title{
DIFFERENT PATTERNS OF ICONIC INFLUENCE IN THE CREATION VS. TRANSMISSION OF THE NICARAGUAN SIGN LANGUAGE LEXICON
}

\author{
ANN SENGHAS ${ }^{* 1}$, JENNIE PYERS $^{2}$, CAROLINE ZOLA $^{1}$ \\ and CHARLOTTE QUINCOSES ${ }^{1}$ \\ *Corresponding Author: asenghas@barnard.edu \\ ${ }^{1}$ Department of Psychology, Barnard College, New York, NY, USA \\ ${ }^{2}$ Department of Psychology, Wellesley College, New York, NY, USA
}

\section{Background}

Observations that iconicity diminishes over time in sign languages (Frishberg, 1975) pose a puzzle - why should something so evidently useful and functional decrease? Perhaps the pattern reflects differences between language-creation and language-transmission processes. If so, the effect might be mediated by the type of iconic representation: iconic signs can depict varied aspects of a referent object, such as how it is handled, or its shape, among other characteristics (Taub, 2001).

We ask, first, whether the prevalence and transparency of iconicity in sign languages results from its recruitment during language creation or during language learning; and second, whether language creation mechanisms favor the same types of iconicity that language-learning mechanisms do.

Existing languages are too mature to address these questions, but a newly emerging sign language in Nicaragua provides an opportunity to see the forces in play at language's earliest stages. Nicaraguan Sign Language (NSL) was created by deaf children and adolescents, starting with an initial cohort who arrived in a new special education school in the 1970s in Managua (Kegl et. al, 1999). Each subsequent age cohort to enter school developed the language further (Senghas \& Coppola, 2001). By comparing the lexicons of different cohorts we can track the changes in iconicity over historical time, as NSL was transmitted to new learners. 


\section{Methods}

As part of a larger study, we compared signs elicited in 2007 and 2017 from a first-cohort signer (35y in 2007) and a second-cohort signer (20y in 2007). From a set of 350 signs for everyday concepts, we selected 64, including 33 concepts rated as high-imageability (650-700) and 31 as low-imageability (200-450; Coltheart, 1981). Signs were categorized according to type of iconicity exhibited (see Fig. 1): either (a) pantomimic (e.g., embodying the referent itself, as in LION, or someone holding the referent, as in SPOON); (b) perceptual (e.g., representing the shape of a referent, as in TURTLE); or (c) arbitrary (e.g., noniconic, as in TRUTH).

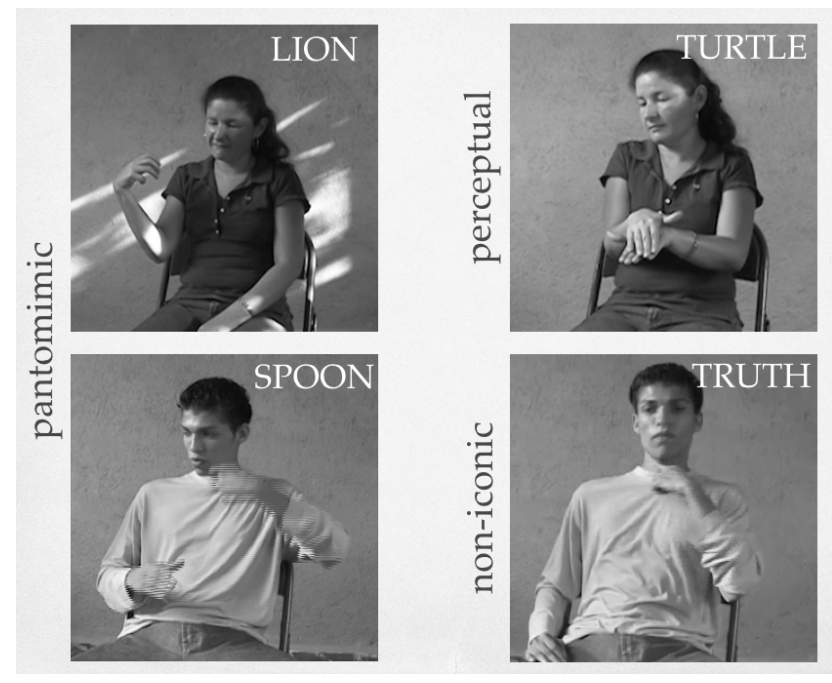

Figure 1. Examples of different types of iconicity in NSL lexical forms.

Three coders, blind to cohort and year, performed paired comparisons of each sign, determining whether any differences between the first- and secondcohort version indicated an increase, decrease, or no change in iconicity. Difference ratings considered both the transparency of iconicity (how easily a mapping could be inferred between the sign and its referent) and the number of articulators included in the iconic mapping (for example, if both the hand and mouth in LION mapped to the body of a lion, as opposed to only the hand). In this way, we compared how signs changed as they were coined and used by their creators over time, vs. passed down to new age cohorts of learners. 


\section{Results}

The overwhelming majority of signs, particularly those for high-imageability concepts, exhibited some kind of iconicity. While the two cohorts' lexicons did not differ in the percentage of iconic signs, they did differ in their distribution of types, with the first-cohort signer producing slightly more pantomimic iconicity, and the second-cohort signer producing slightly more perceptual iconicity. Cross-cohort 2007 comparisons reveal that more signs decreased in iconicity $(45 \%)$ than increased (14\%), with pantomimic signs decreasing the most. Iconicity did not decrease overall from 2007 to 2017 (see Fig. 2).

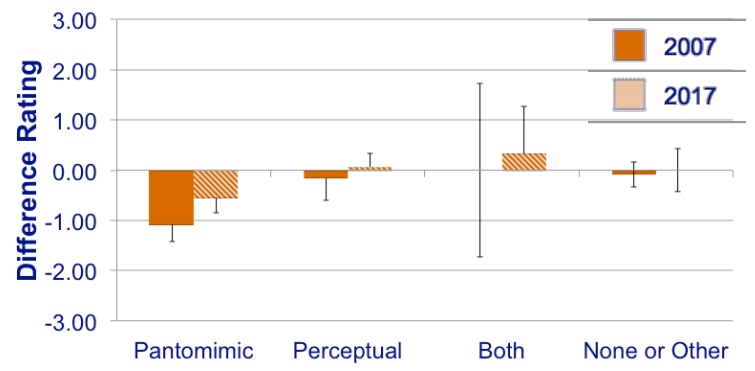

Figure 2. Iconicity differences between cohorts in 2007 and 2017, by type of iconicity. A negative difference rating indicates that the second cohort sign was less iconic than the first cohort sign. In 2007, second cohort signs were less iconic than first, specifically for pantomimic signs. This suggests that pantomimic iconicity decreased as the second cohort acquired NSL in the 1980s. The pattern remains, though attenuated, in 2017.

\section{Discussion}

The high rate of pantomimic iconicity in the first-cohort lexicon reflects its high rate of recruitment during language creation. However, this type of iconicity also appears to be most subject to change, and was compromised during transmission to new learners. Accordingly, perceptual iconicity dominates in the second-cohort lexicon, suggesting that language-learning mechanisms may favor perceptual iconicity. We will consider differences between vertical and horizontal transmission processes, including visual, motoric, learning, and communication pressures, which may conspire to drive the asymmetry in iconicity type in sign language emergence. 


\section{Acknowledgements}

Research funding provided by the James S. McDonnell Foundation (J.P.), Wellesley College Summer Science Center Research Program (J.P.), Barnard College Summer Research Institute (C.Z. \& C.Q.), and National Institutes of Health/NIDCD grant R01 DC005407 (A.S.).

\section{References}

Coltheart, M. (1981). MRC Psycholinguistic Database User Manual: Version 1. Frishberg, N. (1975). Arbitrariness and iconicity: Historical change in American Sign Language. Language, 696-719.

Kegl, J., Senghas, A., \& Coppola, M. (1999). Creation through contact: Sign language emergence and sign language change in Nicaragua. In M. DeGraff (Ed.) Creolization, Diachrony and Development, 179-237.

Senghas, A., \& Coppola, M. (2001). Children creating language: How Nicaraguan Sign Language acquired a spatial grammar. Psychological Science, 12(4), 323-328.

Taub, S. F. (2001). Language from the Body: Iconicity and Metaphor in American Sign Language. Cambridge: Cambridge University Press. 